The diagnosis of thrombocytopenia in newborns and infants is relatively difficult and it should be diagnosis by exclusion. The most important causes of thrombocytopenia that need to be considered in this group are infections, including sepsis as well as CMV infection, fetal hypoxia, chromosomal abnormalities, bone marrow proliferative diseases and neonatal immune thrombocytopenia.

A rare cause of infant thrombocytopenia is genetically determined, most often X-linked, primary immunodeficiency Wiskott-Aldrich syndrome (WAS).

Depending on the type of mutation in the WAS gene, encoding the WASP protein, there is a wide spectrum of clinical phenotypes: X-linked thrombocytopenia, X-linked neutropenia and classic WAS. The last one is characterized by a triad of symptoms: thrombocytopenia, usually with small platelets, recurrent infections, and eczema.

This case - report presents the case of a 4-month-old boy with thrombocytopenia resistant to treatment found from the second day of his life, who was diagnosed with full-blown Wiskott - Aldrich syndrome in the following weeks. An additional difficulty in the differential diagnosis in this case was the boy's negative family history and the normal size of the platelets in the tests, which is atypical for WAS. The diagnosis was confirmed by a molecular test which revealed a new mutation, not registered in the ClinVar and HGMD database. The boy's severe course of disease has been brought under control thanks to a bone marrow transplant from his mother in the age of 12 months.

\section{CENTRAL VENOUS CATHETER FRAGMENTATION IN BOY WITH HEMOPHILIA A AND INHIBITORS}

M Jelić, D Šarić, L Mucavac, S Dejanović-Bekić, M Pavlović, D Turudić, E Bilić. University Hospital Centre Zagreb

\subsection{6/archdischild-2021-europaediatrics.303}

Hemophilia A is characterized by deficiency in FVIII and approximately $30 \%$ of people with severe hemophilia $\mathrm{A}$ are affected by inhibitors.

A 11-year-old boy was diagnosed with severe hemophilia A immediately after birth due to hematoma around right kidney and positive family history. He developed inhibitors on FVIII when he was 12 months of age and therapy was changed in activated prothrombin complex on demand. During next 9 years, recurrent bleeding in the both knees started to occur which was manifested with haemophilic arthropathy.

After several attempt, immune tolerance induction (ITI) was approved by insurance company when he was 10 years old. We started with plasma-derived F VIII in dosage of 100 i.u. per $\mathrm{kg}$ BW daily. Titer of inhibitors was negative after 3 months of ITI, and half life of FVIII was normalised after 6 months.

For ease of administering therapy, central venous catheter (CVC) was inserted in right subclavian vein. On the other hand, usage of CVC can be complicated by infections and thrombosis. One year after insertion of CVC, he became febrile and microbiology findings revealed that blood culture was contaminated with Staphylococcus aureus, Acinetobacter ursingii, Achromobacter xyloxidans and Fusarium proliferatum which was indication for emergency removal of CVC. Unfortunately, during that surgical intervention, breakage of a
CVC occurred and the tip of the catheter was notably missing.

Emergency MSCT of thorax showed tubular structure located in right ventricle with loop at the entrance of right atrium and descending into the inferior vena cava as well as confluence with hepatic veins. To avoid major surgery, the retained portion was successfully removed by the interventional cardiologist through a femoral vein. During procedure, patient received plasma-derived FVIII to maintain the value of FVIII above $80 \%$ and there was no bleeding. Prophylactic antibiotics (meropenem, teicoplanin, liposomal amphotericin B) were continued for 10 days. After three negative blood cultures, negative galactomannan (GM) and 1,3- $\beta$-d-glucan (BDG) tests, he was released home and regularly controlled in day hospital. He is now using plasma-derived F VIII 1500 IU every other day as a prophylaxis of hemophilia $\mathrm{A}$ and is doing well without bleeeding and development of inhibitors.

\section{THE CASE PRESENTATION THE DIAMOND BLACKFAN ANAEMIA CAN BE PRESENTED AS THE PARVOVUIRUS INFECTION}

Liza Collins*, Saadia Rao, Elena Cattaneo, Ramona Onita. Cambridge University Hospital

10.1136/archdischild-2021-europaediatrics.304

Background Diamond-Blackfan Anaemia (DBA) is a congenital type of anaemia characterised by pure red cell aplasia and associated with congenital bone abnormalities. It is chronic macrocytic-normocytic anaemia.

DBA is a heterogeneous genetic disease, inherited as an autosomal dominant inheritance in 40 to $45 \%$ of cases. The remainder 55 to $60 \%$ of cases typically present sporadically.

Case We are introducing the girl who initially was presented with pallor and tachycardia 2 months old. She had $\mathrm{Hb}$ 26g/L. Platelets 973 109/L, neutrophils 0.8 109/L, Reticulocyte 9.5 109/L Blood film showed modest aniso/poikilocytosis, unremarkable WBC, thrombocytosis, no polychromasia.

The flow cytometry performed, was showing:- 65\% lymphocytes,- no blast cells. She was transfused, her $\mathrm{Hb}$ increased to $66 \mathrm{~g} / \mathrm{L}$ However she required several blood transfusions due to low $\mathrm{Hb}$ in 2 months. She had positive IgG for B19 parvovirus infection She initially was diagnosed with transitional aplastic anaemia due to B19 infection However as part of the investigations the elevated level of adenosine deaminase was found. She is currently awaiting the genetic test result, confirming the DBA.

Conclusion It is significantly important to complete the investigation for the DBA if the child presented with aplastic anaemia. Diamond-Blackfan anaemia is a rare disease that carries significant morbidity and mortality if not diagnosed early and managed appropriately.

\section{LOW GRADE GLIOMA BRAF V600E INHIBITOR MONOTHERAPY IN A 12-YEAR OLD CHILD}

Filip Jadrijevic Cvrlje*, Hrvoje Jednacak, Nada Rajacic, Tonci Grmoja, Ana Tripalo Batos, Jasminka Stepan Giljevic. Department of hematology and oncology "Dr Mladen Cepulic', Children's Hospital Zagreb

10.1136/archdischild-2021-europaediatrics.305 